\title{
N-Acetyl Cysteine (NAC) in the Treatment of Obsessive-Compulsive Disorder: A 16-week Double-Blind, Randomised, Placebo-Controlled Study
}

\author{
Jerome Sarris MHSc, PhD ${ }^{1,2}$ (jsarris@unimelb.edu.au), \\ Georgina Oliver $\mathrm{MSc}^{1}$ (georgina.oliver@unimelb.edu.au), \\ David A Camfield PhD ${ }^{2,6,7}$ (camfield@uow.edu.au), \\ Olivia M Dean PhD ${ }^{1,3,5}$ (olivia.dean@barwonhealth.org.au), \\ Nathan Dowling ${ }^{1}$ MPsych (Clin) (ndowling@unimelb.edu.au) \\ Deidre J Smith BSc (Hons) ${ }^{1}$ (djsmit@unimelb.edu.au) \\ Jenifer Murphy BPsych (Hons) ${ }^{1}$ (Jenifer.murphy@unimelb.edu.au) \\ Ranjit Menon MD ${ }^{1}$ (drrmenon@unimelb.edu.au) \\ Michael Berk MD ${ }^{1,3,4,5}$ (MIKEBE AT BarwonHealth.org.au), \\ Scott Blair-West MBBS ${ }^{1}$ (sbwaa@bigpond.com) \\ Chee H Ng MD¹ (cng@unimelb.edu.au)
}

1 The University of Melbourne, Department of Psychiatry, The Melbourne Clinic, Melbourne, Victoria, Australia

2 Centre for Human Psychopharmacology, Swinburne University, Melbourne, Victoria, Australia

3 IMPACT Strategic Research Centre, School of Medicine, Deakin University, Barwon Health PO Box 281 Geelong, Victoria, Australia.

4 Orygen, The National Centre of Excellence in Youth Mental Health and the Centre for Youth Mental Health 35 Poplar Road, Parkville, Melbourne, Victoria, Australia.

5 Florey Institute for Neuroscience and Mental Health, Kenneth Myer Building, Royal Parade, Parkville, Melbourne, Victoria, Australia.

6 Illawarra Health and Medical Research Institute, New South Wales, 2522, Australia7 School of Psychology, University of Wollongong, Wollongong, New South Wales, Australia.

Word Count: 2800

\section{Contact Information:}

Dr Jerome Sarris

The Melbourne Clinic, Department of Psychiatry The University of Melbourne

2 Salisbury St, Richmond, 3121, Melbourne, Australia

Ph:+61394209350 E: jsarris@unimelb.edu.au

Running Header: NAC in the Treatment of OCD 


\begin{abstract}
Background: Obsessive-compulsive disorder (OCD) is a disabling mental illness for which pharmacological and psychosocial interventions are all-to-often inadequate. Recent preclinical and clinical studies have implicated dysfunction of glutamatergic neurotransmission in the pathophysiology of OCD. The amino acid-based nutraceutical $\mathrm{N}$-acetyl cysteine (NAC) is a safe and readily available agent that has been found to modify the synaptic release of glutamate in subcortical brain regions via modulation of the cysteine-glutamate antiporter. Objective: To assess the efficacy and safety of NAC in treating OCD. Methods: A 16-week double-blind, placebocontrolled, randomised trial using 3 grams per day of NAC (1.5 grams 2xday) in 44 participants (aged 18-70) with DSM-5-diagnosed OCD, during 2013-2015. The primary outcome measure was the Yale-Brown Obsessive Compulsive Scale (YBOCS), conducted every four weeks. Results: Analysis of the full sample (Intention-To-Treat) with repeated-measures mixed linear modelling revealed a non-significant time $x$ treatment interaction for the YBOCS scale total score $(p=0.39)$. A per protocol analysis removing protocol violators also failed to show a significant time $x$ treatment interaction for YBOCS total score $(p=0.15)$. However, a significant time $\mathrm{x}$ treatment interaction was observed for the YBOCS "Compulsions" sub-scale in favour of NAC ( $p=0.013)$, with a significant reduction observed at week 12 (dissipating at week 16). At 16-weeks only four (20\%) of the participants were considered "responders" (YBOCS $\geq 35 \%$ reduction at endpoint) versus four (27\%) in the placebo group. The NAC was well tolerated, aside from more cases of heartburn occurring compared to placebo $(p=0.045)$. Conclusion: Further research involving NAC for OCD may require larger samples to detect moderate or small effect sizes, involve dosage or formulation differences, use in concert with exposure therapy, or an additional post-study observational period to mitigate study withdrawal.
\end{abstract}

\title{
Trial Registry: ACTRN 12613000310763
}

\section{Key Points:}

- A 16-week double-blind, randomised, controlled trial was conducted using $3 \mathrm{~g}$ per day of $\mathrm{N}$ acetyl cysteine (NAC) in obsessive-compulsive disorder (OCD).

- A reduction of OCD symptoms occurred over the first 12 weeks in favour of NAC over placebo.

- However, no significant treatment difference was present at week 16 (study endpoint). 
- A significant time $x$ treatment interaction was observed for the YBOCS "Compulsions" subscale in favour of NAC, with a significant reduction observed at week 12, however, this also dissipated at week 16. 


\section{BACKGROUND}

Obsessive-compulsive disorder (OCD) is a disabling mental illness that affects approximately $2 \%$ of the population [1], and for which pharmacological and psychosocial interventions are all-to-often inadequate. A growing body of evidence suggests that abnormalities within the cortico-striatothalamic-cortical (CSTC) circuits are key factors underlying the pathophysiology of OCD $[2,3]$. Specifically, neuronal hyperactivity within the orbitofrontal cortex and ventromedial striatum has been associated with OCD behaviour [4-7]. Glutamate is the primary neurotransmitter within the CSTC, and while not currently conclusive, evidence suggests that disruptions in glutamate neurotransmission are apparent in patients with OCD [8-10]. Significantly higher levels of glutamate have been found in the cerebral spinal fluid and various regions of the brain including the right caudate and orbitofrontal cortex of OCD patients [11-13]. High levels of glutamate are known to elicit oxidative stress [14], and increased oxidative stress has been detected in serum samples from those diagnosed with OCD [15-17] appearing to correlate with symptom severity [18].

The amino acid-based nutraceutical $\mathrm{N}$-acetyl cysteine (NAC) is a safe and readily available agent that has been found to modify the synaptic release of glutamate in subcortical brain regions via modulation of the cysteine-glutamate antiporter [19]. Oral consumption of NAC leads to increases in the brain of extracellular concentrations of cystine (the disulphide derivative of cysteine). NAC acts as a pro-drug for cysteine which has the ability to normalise glutamate transmission in cortico-striatal brain circuitry (in addition to being a key precursor in the synthesis of the endogenous antioxidant glutathione)[20].

Several clinical trials have been conducted using NAC for a range of psychiatric disorders [21, 22], and it has been demonstrated to be well-tolerated in doses up to $8 \mathrm{~g} /$ day. In a review of over 46 placebo-controlled trials for a range of conditions involving 4000 people, orally administered NAC was found to have few significant adverse effects [23]. In respect to OCD-related disorders with similar neurobiology to $O C D$, a clinical trial studied the use of NAC in trichotillomania, an OCDrelated disorder marked by compulsive hair pulling. The 12-week, double-blind, randomised controlled trial $(\mathrm{RCT})(n=50)$ revealed that NAC $(1.2 \mathrm{~g}-2.4 \mathrm{~g} /$ day) significantly reduced symptoms on the Massachusetts General Hospital Hair Pulling Scale, and the Psychiatric Institute 
Trichotillomania Scale [24]. Fifty-six percent of participants were "much or very much improved" in the NAC group, compared with $16 \%$ taking placebo $(p=0.003)$. Furthermore, NAC has also been found to ameliorate symptoms in a number of other impulsive and compulsive behaviours such as pathological gambling [25] and compulsive nail biting [26].

While NAC has evidence of efficacy in bipolar depression, schizophrenia, trichotillomania and other compulsive behaviours, to our knowledge there is only pilot data to date assessing its efficacy in treating OCD. Recent data exists from a double-blind RCT of adjunctive NAC in the treatment of OCD [27]. This 12-week Iranian study involved 48 participants with DSM-IV diagnosed $\mathrm{OCD}$, using $2.4 \mathrm{~g} /$ day of NAC or matching placebo tablets. The NAC treatment group demonstrated a significant effect over placebo for ameliorating OCD symptoms on the Yale-Brown Obsessive Compulsive Scale (YBOCS; $p=0.003$ ). A gradual and continual decrease in symptom severity was evident from week-4, with NAC showing significance over placebo from week-8 onwards [27].

Although a promising result, there are two important study characteristics to note. First, the effect of NAC over placebo only began to separate towards the end of the study. Second, a dose of $2.4 \mathrm{~g} /$ day was used. Recent data from clinical trials involving bipolar [28] and schizophrenia patients [29] have shown that longer treatment times using NAC may be needed. In addition a study on ADHD showed a reduction of symptoms using a higher NAC dose, querying the optimal dose of the agent [30]. This raises the possibility that NAC may be more effective in a longer clinical trial or when using a higher dose. Due to this, and to independently replicate the Afshar study [27], we present the results of our 16-week study using $3 g$ per day of NAC to treat OCD.

\section{METHODS}

\subsection{Overview}

This is a 16-week double-blind RCT using $3 \mathrm{~g} /$ day of NAC ( $1.5 \mathrm{~g} 2$ xday) or matching placebo in 44 randomised adult participants with DSM-5-diagnosed OCD. The study was conducted, according to Good Clinical Practice, at The Melbourne Clinic (where participants were primarily recruited from) during June 2013 to April 2015. The study is registered on ANZCTR https://www.anzctr.org.au/Trial/Registration/TrialReview.aspx?id=363797, and has ethics approval from The Melbourne Clinic Ethics Committee (TMC_HREC no.219). 


\subsection{Inclusion criteria}

Participants were required to meet the following criteria: aged 18-70 and have the capacity to consent to the study and follow its procedures; fulfil the DSM-IV-TR diagnostic criteria (based on the Mini International Neuropsychiatric Interview-plus; MINI-plus) for Obsessive Compulsive Spectrum Disorder (OCSD; and diagnosed OCD with the insight specifier from the DSM-5, via clinician assessment), and be scoring 16 or over on the YBOCS at the time of study entry; be on either no treatment or a stable treatment regimen for a minimum of four weeks of current treatment and a minimum of 12 weeks if this is their first OCD treatment. Self-reported OCD was assessed and confirmed by physician diagnosis; female participants were required to use effective contraception if of childbearing age and sexually active.

\subsection{Exclusion criteria}

Participants were ineligible to enter the trial if they had any of the following conditions: currently engaged in a psychological program (e.g. a 2-week OCD behavioural modification program or an intensive cognitive behavioural therapy course [ongoing psychological or medical care was accepted]); known or suspected clinically unstable serious medical disorder; bipolar I disorder, schizophrenia, epilepsy, asthma or recent gastrointestinal ulcers; pregnancy or breastfeeding; consumption per day of over $250 \mathrm{mg}$ of NAC, $100 \mathrm{mcg}$ of selenium, or $500 \mathrm{IU}$ of vitamin $\mathrm{E}$; history of hypersensitivity or intolerance to NAC or any component of the preparation.

\subsection{Assessments}

The primary outcome assessment was the YBOCS [31], which is the gold-standard clinician-rated scale for assessing obsessive and compulsive symptoms. Secondary outcome assessments were the Clinical Global Impression Scales- severity and improvement (CGI-S, CGI-I)[32], to measure general clinician-rated improvement or worsening of OCD; the Hamilton Anxiety Rating Scale[33] to measure changes in anxiety levels; the Montgomery-Asberg Depression Rating Scale[34] to measure changes in depression levels; and the General Health Questionnaire (GHQ-28)[35] to assess any effects on general health, and the subdomains of depression, anxiety, social impairment, and hypochondriasis; and purpose-designed demographics/medical history/drug/alcohol use forms. 


\subsection{Intervention}

Randomised individuals undertook a specific dosing schedule (Table 1). The NAC was provided in opaque capsule form, produced by Lipa Pharmaceuticals PTY Ltd., and supplied by Bioceuticals PTY Ltd., in strict compliance with pharmaceutical Good Manufacturing Procedure. The certificate of analysis confirmed the NAC had a $99.97 \%$ purity. The placebo group was given the same dosing regimen of matching opaque capsules containing cellulose.

Insert Table 1 about here

\subsection{Randomisation and Blinding}

Randomisation of participants to treatment groups was determined by computerised random allocation. Recruited eligible participants were assigned a participant number and allocated capsule packs accordingly. Blinding was achieved by enlisting a person outside of the project to code the treatments and maintain the key to this code until data collection was completed. The randomisation code was set up in a $3 \times 2$ block design (e.g. AABABABBBAAABB), and participants were allocated sequentially. In addition, a capsule audit was conducted by an independent researcher who was not involved with data collection (to avoid any unblinding via potential detection of NAC sulphur scent by the researcher assessing participants). A $75 \%$ tablet consumption rate was considered to be minimum threshold for compliance.

\subsection{Data analysis}

We aimed to recruit a sample size of 50 participants ( 25 participants in each arm), with a projected attrition rate of $10 \%$. The study was powered to detect a potential moderate interaction $(f=0.15$ effect size) between treatment group and time (using all post-baseline data via intention-to-treat analysis and a repeated measures Analysis of Variance: ANOVA). The primary efficacy analysis assessed treatment $x$ study visit differences for the primary outcome measure (YBOCS)[31] using a 
mixed-effects linear model, with an unstructured covariance structure fitted to the within-subjects (repeated) study visit variable. All tests of treatment effects were conducted using a two-tailed alpha level of 0.05 and 95\% confidence intervals. Data were analysed via SPSS 22.0.

\section{FIGURE 1 and TABLES 2,3 ABOUT HERE}

\section{RESULTS}

\subsection{Participant Characteristics}

From 189 participants screened, a total of 44 participants met DSM-IV and DSM-5 criteria for OCSD/OCD, respectively, and were randomised into the study (Figure 1). As detailed in Tables 2 and 3, there were no statistically significant imbalances of any demographic features of the sample, including for compliance. In concordance with previous epidemiological studies [36], the majority of participants were male $(n=24 ; 54.5 \%)$. The mean age of the sample was 37 years (range 18 to 68 years). The chronicity of OCD ranged from two years to over 45 years in duration $(M=16.36$ years \pm 9.5 years). Five participants $(11.4 \%)$ reported a family history of OCD and over half the participants (56.8\%; $n=25$ ) had a DSM-IV diagnosed co-morbid psychiatric disorder. The majority of participants (70.5\%) were taking psychotropic medication, namely selective serotonin re-uptake inhibitors (56.8\%) and 13 participants $(29.5 \%)$ had adjunctive therapies such as antipsychotics. See Tables 2 and 3 for more demographic data. Per-protocol analysis was completed on 35 participants, with nine removed due to: $<75 \%$ compliance ( $n=6 ; 4$ placebo and 2 NAC; NS), no follow-up data $(n=2)$, and false reporting $(n=1)$. There were no significant differences between groups for any characteristics for the per protocol group (data not shown).

\subsection{Primary Outcome}

An initial analysis for the total sample $(n=44$ : NAC $(n=22)$, placebo $(n=22))$ revealed a nonsignificant interaction $\left(F_{4,144.63}=1.15, p=0.39\right)$ between treatment and time, with the NAC group having a reduction from baseline to week 16 of 4.78 points $(26.55 \pm 5.67$ to $21.77 \pm 8.04)$ on the YBOCS, compared to 4.50 points $(25.55 \pm 4.85$ to $21.05 \pm 9.31)$ for the placebo group (Table 4). Similarly, for the per protocol analysis $(n=35)$ no significant time by treatment interaction was found, with NAC $(n=20)$ not differing from placebo $(n=15)$ for reduction of OCD symptoms on the YBOCS over 16 weeks $\left(F_{4,30.668,}=1.83, p=0.15\right.$; Figure 2$)$. The YBOCS total score 
from baseline to week 16 for NAC was $25.65 \pm 5.06$ to $20.23 \pm 8.86$ (5.42 point reduction) compared to $25.87 \pm 4.70$ to $20.82 \pm 8.29$ (5.05 point reduction) for placebo. While a difference in symptom reduction was evident between groups at week 12 of 3.18 points, this was not significant. However, a significant treatment $x$ study visit interaction was observed for the YBOCS “Compulsions" sub-scale $(n=35)$ in favour of NAC (Figure 3; $F_{4,27.85}=3.861, p=0.013$ ), with a significant reduction from baseline to week 12 observed in the NAC group $(p<0.001)$ that was not observed in the placebo group. However, this difference had dissipated at week 16. In contrast, on the "Obsessions" subscale, no significant effect was found $\left(F_{4,30.79}=0.48, p=0.75\right)$. At 16-weeks only four (20\%) of the participants in the NAC group were considered "responders" (YBOCS $\geq 35 \%$ reduction at endpoint) versus four $(27 \%)$ in the placebo group. OCD severity, or current treatment did not alter the results. When stratified for severity, gender, comorbidities, and chronicity, there was no statistically significant difference between NAC and placebo on the YBOCS scale total score. Likewise, investigations into whether NAC was effective for differing presentations of OCD (e.g. contamination, sexual/religious, symmetry/ordering obsessions), also revealed no significant effects (although this was unlikely due to the small sub-samples analysed). A sub-analysis of participants taking medication (70.5\%; primarily selective serotonin reuptake inhibitors) also revealed no significant result.

\subsection{Secondary Outcomes}

No significant result was revealed for any of the secondary outcomes on anxiety levels (HAMA), depression levels (MADRS), Clinical Global Impression of change/improvement (CGI-S/I), or general health (GHQ-28); see Table 4. The NAC medication was well-tolerated with no differences in the total adverse effects (AEs) between the groups (14 minor AEs in the placebo group compared to 15 in the active group; $\left.X^{2} 0.16, p=0.90\right)$, or withdrawals due to any serious adverse effects. The only clinical AE difference of note was four cases of heartburn occurring in the NAC group compared to none in the placebo group $\left(X^{2} 4.02, p=0.045\right)$.

Insert Table 4 and Figures 2,3 about here

\section{DISCUSSION}

Although our study employed a higher dose and a longer treatment period of adjunctive NAC in OCD than the Afshar pilot study[27], it did not confirm their positive findings. While a 
significant effect for NAC over placebo occurred at week 12 (for compulsions) in our study, however this dissipated by week 16, raising the question of a type 2 error. Regardless, this interesting finding is of value, and encourages further exploration into whether NAC may have a preferential effect on the compulsivity aspect of OCD. The reasons for this effect (if not an artefact of chance) are unclear, however this may be due to the neurobiology/neurocognition of compulsivity. The underlying nature of this potential relationship may reflect the effect of NAC on compulsive behaviour, as it has previously been found to be effective in gambling [25], and substance abuse [37]. This maybe mediated through the glutamate pathway modulation, and in part by reduction of oxidative stress [21].

Our recent systematic review exploring NAC for OCD and OCD-related disorders (OCRD) [38] found that the balance of evidence (mainly case studies) supported the use of NAC in presentations of moderate-severity OCD and OCRD. Doses of $2.4 \mathrm{~g}-3 \mathrm{~g} / \mathrm{day}$ for a minimum of eight weeks were considered adequate for exerting an initial therapeutic effect. However, even within these patient groups, response rates are variable, and some reports, including our present study, did not confirm efficacy. In addition, previous data predominantly focused on NAC being used for various OCD-related disorders including trichotillomania and excoriation, and in paediatric populations, and thus may not reflect the activity in adult OCD. NAC is yet to be investigated as a monotherapy, and whether its effects are enhanced or reduced in the presence of pharmacotherapies is unknown. The $3 \mathrm{~g}$ of NAC used in the study appears to be well-tolerated, with minimal adverse effects reported. Mild gastrointestinal effects appear to be the most common symptom in previous studies, and this was noted in our study with four cases of heartburn reported in the NAC group compared to none in the placebo group (potentially due to the irritant effect of the sulphur-based compound).

We identify several limitations to the current study. Firstly, the sample size was modest, and a larger sample may have been powered to detect a smaller effect. It is challenging to recruit an OCD population, and a previous NAC OCD RCT conducted by Pittenger from Yale, was abandoned due to recruitment challenges [38]. Interestingly, several participants were difficult to rate on the YBOCS due to severity and complexity of illness, and obsessive "scrupulosity" affecting reporting fidelity. Another limitation was that while the study included people with OCD who were on "treatment as usual", potential confounding presented with participants 
taking different medications, psychological therapies, or no current active treatment. While sub-analyses attempted to ascertain any difference between treatments, the study was not adequately powered to assess this. The efficacy of NAC may be influenced by the presence of concomitant psychotherapy or pharmacotherapy, and it is generally harder to demonstrate an effect as add-on therapy than monotherapy. Further, it is possible that a longer treatment period may be justified, with NAC potentially requiring over 16 weeks to be effective. Studies of patients with NAC in schizophrenia and bipolar disorder needed 6 months for the full effect to be evident. The 16-week study period also did not reflect the duration of full dose due to the titration phase. Regardless, we cannot discount tolerance or loss of effect occurring by week 12.

\section{CONCLUSIONS}

Results of a 16-week double-blind RCT using $3 g$ per day of N-acetyl cysteine (NAC) in OCD, revealed a reduction of OCD symptoms occurred over the first 12 weeks in favour of NAC over placebo. This main result however was non-significant, and no significant treatment difference was present at week 16 (study endpoint). A significant treatment $\mathrm{x}$ time interaction was observed for the YBOCS "Compulsions" sub-scale in favour of NAC, with a significant reduction observed at week 12, however this dissipated at week 16. Future studies on NAC may involve continued exploration the pharmacokinetics and pharmacodynamics to elucidate more specific therapeutic indications for the agent within different clinical presentations of OCD and OCRD. Future clinical trials which incorporate Research Domain Criteria (RDoC) concordant target engagement measures to determine glutamate abnormalities and response to NAC, such as magnetic resonance spectroscopy or function magnetic resonance brain imaging would help address whether NAC has the ability to directly modulate activity in the regions most heavily implicated in the pathogenesis of these disorders. Further research work may also involve dosage or formulation differences, use in concert with exposure therapy, a longer study period, or an additional post-study observational period to mitigate any study withdrawal effect.

ACKNOWLEDGEMENTS: The NAC was kindly donated and supplied by Bioceuticals ${ }^{\circledR}$. IS is funded by a CR Roper Fellowship. MB is supported by a NHMRC Senior Principal Research Fellowship 1059660. 


\section{COMPLIANCE WITH ETHICAL STANDARDS:}

This study was given ethics approval by The Melbourne Clinic Ethics Committee (TMC_HREC no.219), which is an National Health and Medical Research Council (NHMRC) registered ethics committee in Australia, and conducted according the 1964 Declaration of Helsinki. All participants freely gave informed consent before commencing the study.

\section{Funding}

No funding was received for the conduct of this study nor for preparation of this manuscript. The NAC and placebo tablets were supplied free of charge by the company Bioceuticals (this company does not currently sell this product; additionally, they had no involvement in study design/conduct/data analysis/publication of results).

\section{Conflicts of interest}

No specific conflicts are identified from any individual author. JS has received honoraria, research support, royalties, or consultancy or travel grant funding from Bioceuticals, Blackmores, Taki Mai, Integria Health Pepsico, HealthEd, Soho-Flordis, Pfizer, Elsevier, the Society for Medicinal Plant and Natural Product Research, CR Roper Fellowship, The NHMRC, Chaminade University. OMD is a Research Fellow and has received grant support from the Brain and Behavior Foundation, Simons Autism Foundation, Australian Rotary Health, Stanley Medical Research Institute, Deakin University, Brazilian Society Mobility Program Lilly, NHMRC and an ASBD/Servier grant. She has also received in kind support from BioMedica Nutraceuticals, NutritionCare and Bioceuticals. $\mathrm{CN}$ has previously received research support from Wyeth and Lundbeck, and speaker honoraria from Servier, Bristol-Myers Squibb, Organon, Eli Lilly, GlaxoSmithKline, Janssen- Cilag, Astra-Zenaca, Wyeth, and Pfizer. MB has received grant/research support from the $\mathrm{NIH}$, Cooperative Research Centre, Simons Autism Foundation, Cancer Council of Victoria, Stanley Medical Research Foundation, MBF, NHMRC, Beyond Blue, Rotary Health, Geelong Medical Research Foundation, Bristol Myers Squibb, Eli Lilly, Glaxo SmithKline, Meat and Livestock Board, Organon, Novartis, Mayne Pharma, Servier and Woolworths, has been a speaker for Astra Zeneca, Bristol Myers Squibb, Eli Lilly, Glaxo SmithKline, Janssen Cilag, Lundbeck, Merck, Pfizer, Sanofi Synthelabo, Servier, Solvay and Wyeth, and served as a consultant to Astra Zeneca, Bioadvantex, Bristol Myers Squibb, Eli Lilly, Glaxo SmithKline, Janssen Cilag, Lundbeck Merck and Servier and is a co-inventor on two 
provisional patents regarding the use of NAC and related compounds for psychiatric indications assigned to the Mental Health Research Institute.

\section{REFERENCES}

1. American Psychiatric Association. Diagnostic And Statistical Manual of Mental Disorders. 5th ed. Arlington: American Psychiatric Association; 2013.

2. Ting JT, Feng G. Neurobiology of obsessive-compulsive disorder: insights into neural circuitry dysfunction through mouse genetics. Current opinion in neurobiology. 2011;21(6):842-8.

3. Harrison BJ, Pujol J, Cardoner N, et al. Brain corticostriatal systems and the major clinical symptom dimensions of obsessive-compulsive disorder. Biological psychiatry. 2013;73(4):321-8.

4. Rotge JY, Guehl D, Dilharreguy B, et al. Meta-analysis of brain volume changes in obsessivecompulsive disorder. Biological psychiatry. 2009;65(1):75-83.

5. Rotge JY, Langbour N, Jaafari N, et al. Anatomical alterations and symptom-related functional activity in obsessive-compulsive disorder are correlated in the lateral orbitofrontal cortex. Biological psychiatry. 2010;67(7):e37-8.

6. Milad MR, Rauch SL. Obsessive-compulsive disorder: beyond segregated cortico-striatal pathways. Trends in cognitive sciences. 2012;16(1):43-51.

7. Ahmari SE, Spellman T, Douglass NL, et al. Repeated cortico-striatal stimulation generates persistent OCD-like behavior. Science. 2013;340(6137):1234-9.

8. Pittenger C, Bloch $\mathrm{MH}$, Williams K. Glutamate abnormalities in obsessive compulsive disorder: neurobiology, pathophysiology, and treatment. Pharmacology \& therapeutics. 2011;132(3):314-32.

9. Wu K, Hanna GL, Rosenberg DR, et al. The role of glutamate signaling in the pathogenesis and treatment of obsessive-compulsive disorder. Pharmacology, biochemistry, and behavior.

2012;100(4):726-35.

10. Kariuki-Nyuthe C, Gomez-Mancilla B, Stein D. Obsessive Compulsive Disorder and the Glutamate System. Curr Opin Psychiatry. 2014;27:32-7.

11.Yucel M, Wood SJ, Wellard RM, et al. Anterior cingulate glutamate-glutamine levels predict symptom severity in women with obsessive-compulsive disorder. The Australian and New Zealand journal of psychiatry. 2008;42(6):467-77.

12. Bhattacharyya S, Khanna S, Chakrabarty K, et al. Anti-brain autoantibodies and altered excitatory neurotransmitters in obsessive-compulsive disorder. Neuropsychopharmacology : official publication of the American College of Neuropsychopharmacology. 2009;34(12):2489-96.

13. Chakrabarty K, Bhattacharyya S, Christopher R, et al. Glutamatergic dysfunction in OCD.

Neuropsychopharmacology : official publication of the American College of Neuropsychopharmacology. 2005;30(9):1735-40.

14. Burdo J, Dargusch R, Schubert D. Distribution of the cystine/glutamate antiporter system xc- in the brain, kidney, and duodenum. The journal of histochemistry and cytochemistry : official journal of the Histochemistry Society. 2006;54(5):549-57.

15. Ersan S, Bakir S, Erdal Ersan E, et al. Examination of free radical metabolism and antioxidant defence system elements in patients with obsessive-compulsive disorder. Prog Neuropsychopharmacol Biol Psychiatry. 2006;30(6):1039-42.

16. Ozdemir E, Cetinkaya S, Ersan S, et al. Serum selenium and plasma malondialdehyde levels and antioxidant enzyme activities in patients with obsessive-compulsive disorder. Progress in neuropsychopharmacology \& biological psychiatry. 2009;33(1):62-5. 
17. Behl A, Swami G, Sircar SS, et al. Relationship of possible stress-related biochemical markers to oxidative/antioxidative status in obsessive-compulsive disorder. Neuropsychobiology. 2010;61(4):210-4 18. Chakraborty S, Singh OP, Dasgupta A, et al. Correlation between lipid peroxidation-induced TBARS level and disease severity in obsessive-compulsive disorder. Progress in neuro-psychopharmacology \& biological psychiatry. 2009;33(2):363-6.

19. Moran MM, McFarland K, Melendez RI, et al. Cystine/glutamate exchange regulates metabotropic glutamate receptor presynaptic inhibition of excitatory transmission and vulnerability to cocaine seeking. The Journal of neuroscience : the official journal of the Society for Neuroscience.

2005;25(27):6389-93.

20.Choy KH, Dean O, Berk M, et al. Effects of $\mathrm{N}$-acetyl-cysteine treatment on glutathione depletion and a short-term spatial memory deficit in 2-cyclohexene-1-one-treated rats. Eur J Pharmacol. 2010;649(13):224-8.

21.Berk M, Malhi GS, Gray $L$, et al. The promise of $\mathrm{N}$-acetylcysteine in neuropsychiatry. Trends in pharmacological sciences. 2013;34(3):167-77.

22. Deepmala, Slattery J, Kumar N, et al. Clinical Trials of $\mathrm{N}$-acetylcysteine in Psychiatry and Neurology: A Systematic Review. Neurosci Biobehav Rev. 2015.

23. Atkuri K, Mantovani J, Herzenberg L. N-Acetylcysteine a safe antidote for cysteine/glutathione deficiency. Current Opinion in Pharmacology. 2007;7(4):355-9.

24.Grant JE, Odlaug BL, Kim SW. N-acetylcysteine, a glutamate modulator, in the treatment of trichotillomania: a double-blind, placebo-controlled study. Arch Gen Psychiatry. 2009;66(7):756-63.

25.Grant JE, Kim SW, Odlaug BL. N-acetyl cysteine, a glutamate-modulating agent, in the treatment of pathological gambling: a pilot study. Biological psychiatry. 2007;62(6):652-7.

26.Ghanizadeh A, Derakhshan N, Berk M. N-acetylcysteine versus placebo for treating nail biting, a double blind randomized placebo controlled clinical trial. Anti-inflammatory \& anti-allergy agents in medicinal chemistry. 2013;12(3):223-8.

27.Afshar $\mathrm{H}$, Roohafza $\mathrm{H}$, Mohammad-Beigi $\mathrm{H}$, et al. $\mathrm{N}$-acetylcysteine add-on treatment in refractory obsessive-compulsive disorder: a randomized, double-blind, placebo-controlled trial. J Clin

Psychopharmacol. 2012;32(6):797-803.

28. Berk M, Copolov DL, Dean O, et al. N-acetyl cysteine for depressive symptoms in bipolar disorder--a double-blind randomized placebo-controlled trial. Biological psychiatry. 2008;64(6):468-75.

29.Berk M, Copolov D, Dean O, et al. Jeavons S, Schapkaitz I, et al. N-acetyl cysteine as a glutathione precursor for schizophrenia--a double-blind, randomized, placebo-controlled trial. Biological psychiatry. 2008;64(5):361-8.

30.Garcia RJ, Francis L, Dawood M, et al. Attention deficit and hyperactivity disorder scores are elevated and respond to $\mathrm{N}$-acetylcysteine treatment in patients with systemic lupus erythematosus. Arthritis and rheumatism. 2013;65(5):1313-8.

31.Goodman W. The Yale-Brown Obsessive Compulsive Scale. Arch Gen Psychiatry. 1989;46:1006-11.

32. Guy W, Bonato R. CGI: Clinical Global Impressions. Manual for the ECDEU Assessment Battery2 Rev ed Chevy Chase, Md: National Institute of Mental Health. 1970(12-1-12-6.).

33. Hamilton M. The assessment of anxiety states by rating. Br J Med Psychol. 1959;32(1):50-5.

34. Montgomery SA, Asberg M. A new depression scale designed to be sensitive to change. $\mathrm{Br} \mathrm{J}$ Psychiatry. 1979;134:382-9.

35.Goldberg D. Manual of the General Health Questionnaire. Windsor, England: NFER Publishing; 1978. 36. Ruscio AM, Stein DJ, Chiu WT, et al. The epidemiology of obsessive-compulsive disorder in the National Comorbidity Survey Replication. Mol Psychiatry. 2010;15(1):53-63.

37.Amen SL, Piacentine LB, Ahmad ME, et al. Repeated $\mathrm{N}$-acetyl cysteine reduces cocaine seeking in rodents and craving in cocaine-dependent humans. Neuropsychopharmacology. 2011;36(4):871-8.

38. Oliver G, Dean O, Camfield D, et al. N-Acetyl Cysteine (NAC) in the Treatment of Obsessive Compulsive and Related Disorders: A Systematic Review. Clinical Psychopharmacology and Neuroscience. 2014; In Press. 
Table 1. Dosing Schedule

\begin{tabular}{cccc}
\hline Week & Dosage & $\begin{array}{c}\text { Tablets per day } \\
(500 \mathrm{mg})\end{array}$ & Dosing regimen \\
\hline 1 & $1000 \mathrm{mg}$ & 2 & 1 morning / 1 evening \\
2 & $2000 \mathrm{mg}$ & 4 & 2 morning / 2 evening \\
3 & $3000 \mathrm{mg}$ & 6 & 3 morning / 3 evening \\
\hline
\end{tabular}

Figure 1. Consort Flowchart

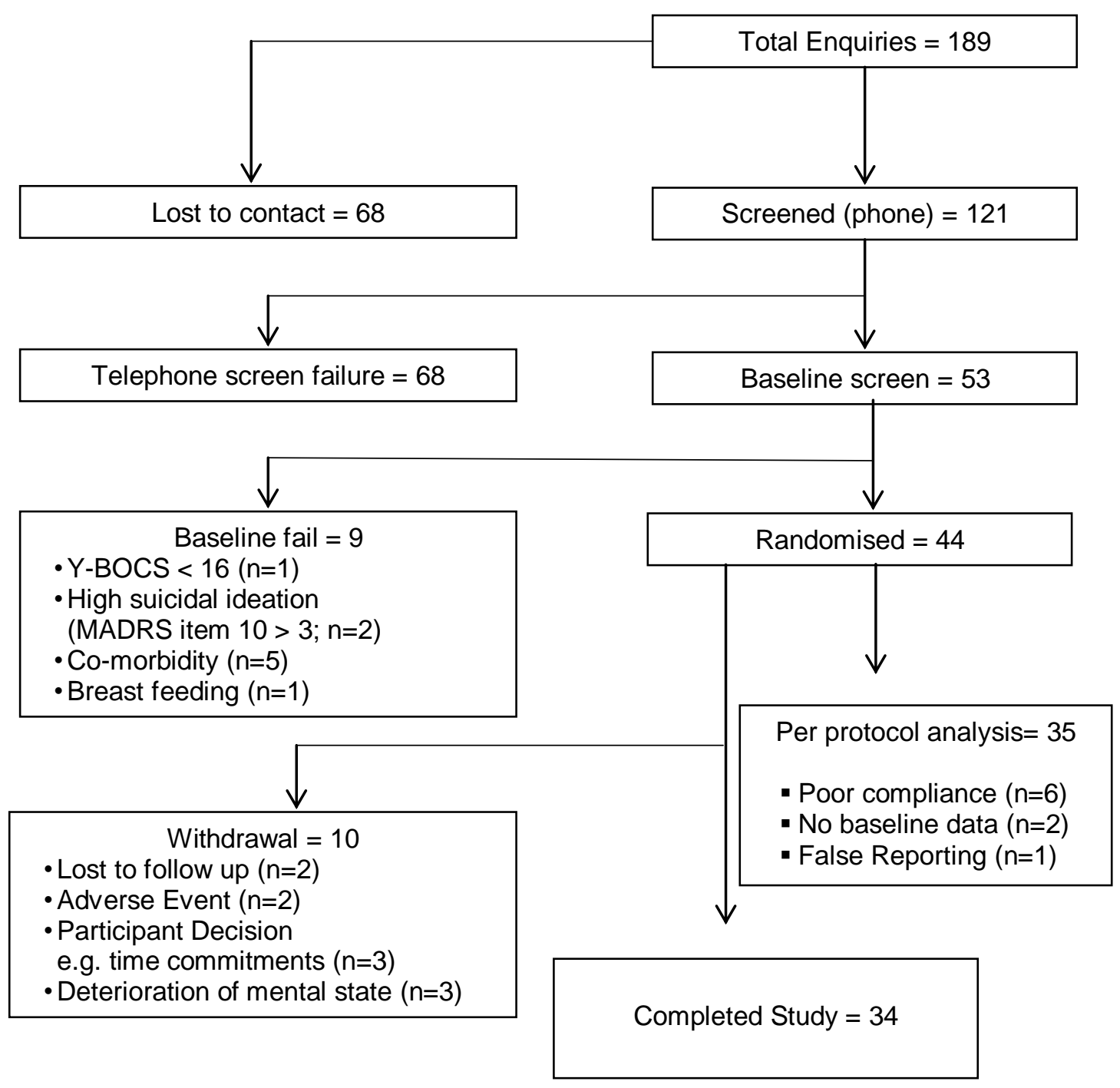


Table 2. Participant Characteristics

\begin{tabular}{|c|c|c|c|c|}
\hline & $\begin{array}{c}\text { NAC }(n=22) \\
\text { frequency(\%)/ } \\
M \pm S D\end{array}$ & $\begin{array}{c}\text { Placebo } \\
(n=22) \\
\text { frequency }(\%) / \\
M \pm S D\end{array}$ & $\begin{array}{c}\text { Test } \\
\text { Statistic } \\
\text { t-test } / \chi^{2}\end{array}$ & $p$-value $[95 \% \mathrm{Cl}]$ \\
\hline \multicolumn{5}{|l|}{ Gender } \\
\hline Male & $12(27.3)$ & $12(27.3)$ & & 1.00 \\
\hline Female & $10(22.7)$ & $10(22.7)$ & & 1.00 \\
\hline Age & $39.14 \pm 12.8$ & $34.86 \pm 11.4$ & -1.17 & 0.37 \\
\hline Marital Status & & & 0.43 & 0.81 \\
\hline Married & $6(27.3)$ & $8(36.4)$ & & \\
\hline Never married & $15(68.2)$ & 13 (59.1) & & \\
\hline Divorced/separated & $1(4.5)$ & $1(4.5)$ & & \\
\hline Education & & & 4.47 & 0.61 \\
\hline Tertiary & $9(40.9)$ & $11(50.0)$ & & \\
\hline High school & $5(22.7)$ & $6(27.3)$ & & \\
\hline Some high school & $5(22.7)$ & $3(13.7)$ & & \\
\hline $\begin{array}{l}\text { Technical } \\
\text { college/Diploma }\end{array}$ & $3(13.7)$ & $2(9.0)$ & & \\
\hline Occupation & & & 2.63 & 0.76 \\
\hline Employed & $12(54.5)$ & $15(68.2)$ & & \\
\hline Unemployed & $10(45.5)$ & $7(31.8)$ & & \\
\hline
\end{tabular}

$\chi^{2}$ : Chi-squared test; $\mathrm{M}=$ Mean; $\mathrm{SD}=$ Standard Deviation; $\mathrm{NAC}=\mathrm{N}$-acetyl cysteine 
Table 3. Clinical Features of Participants at Baseline

\begin{tabular}{lcccc}
\hline Measure & $\begin{array}{c}\text { NAC } \\
(n=22)\end{array}$ & $\begin{array}{c}\text { Placebo } \\
(n=22)\end{array}$ & $\begin{array}{c}\text { Test Statistic } \\
\text { t-test } / \chi^{2}\end{array}$ & p-value [95\% Cl] \\
\hline YBOCS & $26.55 \pm 5.67$ & $25.55 \pm 4.85$ & -0.63 & 0.75 \\
\hline OCD Severity* & & & 0.00 & 1.00 \\
\hline Mild & 2 & 2 & & \\
Moderate & 16 & 16 & & \\
Severe & 4 & 4 & & 0.94 \\
OCD chronicity (years) & $17.59 \pm 8.94$ & $15.14 \pm 10.1$ & -0.85 & 0.19 \\
\hline Family history of OCD & 1 & 4 & 1.73 & 0.76 \\
\hline Medication+ & & & & 1.00 \\
\hline SSRI & 13 & 12 & 0.09 & 0.43 \\
SSNRI & 2 & 2 & 0.00 & 0.55 \\
Antipsychotic & 3 & 5 & 0.61 & 0.71 \\
TCA & 2 & 1 & 0.36 & 0.31 \\
GABA agents & 5 & 4 & 0.14 & 1.02 \\
Glutamate antagonist & 0 & 1 & 2.73 & \\
Monotherapy & 4 & 9 & & \\
\hline
\end{tabular}

OCD: Obsessive-Compulsive Disorder; NAC: N-acetyl cysteine; YBOCS: Yale-Brown Obsessive Compulsive Scale; GABA: Gamma-amino butyric acid; SSNRI: Selective serotonin-noradrenaline reuptake inhibitor; SSRI: Selective serotonin reuptake inhibitor; TCA: Tricyclic antidepressant; $\chi^{2}$ :Chi-squared test;

${ }^{*}$ OCD severity determined by YBOCS score of $<20=$ mild; $20-30=$ moderate; $>30=$ severe; + : Some participants were taking multiple medications 
Table 4. Results

N-Acetyl Cysteine

Placebo

\begin{tabular}{|c|c|c|c|c|c|c|c|c|}
\hline & \multicolumn{2}{|c|}{ Baseline } & \multicolumn{2}{|c|}{ Week 16} & \multicolumn{2}{|c|}{ Baseline } & \multicolumn{2}{|c|}{ Week 16} \\
\hline & ITT & PP & ITT & PP & ITT & $\mathrm{PP}$ & $\mathrm{ITT}$ & PP \\
\hline YBOCS Total & 26.55 (5.67) & $25.65(5.06)$ & $21.77(8.04)$ & $20.23(8.86)$ & $25.55(4.85)$ & $25.87(4.70)$ & $21.05(9.31)$ & $20.82(8.29)$ \\
\hline $\begin{array}{l}\text { YBOCS } \\
\text { Obsessions }\end{array}$ & $13.27(3.31)$ & $12.80(2.91)$ & $10.45(4.24)$ & $9.90(4.11)$ & $12.73(2.83)$ & $12.73(2.94)$ & $10.59(4.55)$ & $10.18(4.14)$ \\
\hline $\begin{array}{l}\text { YBOCS } \\
\text { Compulsions } \\
+\end{array}$ & $13.27(2.91)$ & $12.85(2.50)$ & $11.32(4.40)$ & $10.71(4.78)$ & $12.81(2.36)$ & $13.13(2.48)$ & $10.45(4.93)$ & $10.32(4.84)$ \\
\hline \multicolumn{9}{|l|}{ HAMA } \\
\hline & \multicolumn{2}{|c|}{$12.73(7.72)$} & \multicolumn{2}{|c|}{$10.00(6.27)$} & \multicolumn{2}{|c|}{$13.55(7.51)$} & \multicolumn{2}{|c|}{$10.05(6.43)$} \\
\hline \multicolumn{9}{|l|}{ MADRS } \\
\hline & \multicolumn{2}{|c|}{$14.45(9.75)$} & \multicolumn{2}{|c|}{$12.27(9.02)$} & \multicolumn{2}{|c|}{$15.41(9.26)$} & \multicolumn{2}{|c|}{12.86 (11.79) } \\
\hline \multicolumn{9}{|l|}{ CGI-S } \\
\hline & \multicolumn{2}{|c|}{$4.45(1.06)$} & \multicolumn{2}{|c|}{$3.95(1.40)$} & \multicolumn{2}{|l|}{$3.96(1.33)$} & \multicolumn{2}{|c|}{$3.60(1.26)$} \\
\hline CGI-I\# & \multicolumn{2}{|c|}{$3.43(0.92)$} & \multicolumn{2}{|c|}{$3.00(1.14)$} & \multicolumn{2}{|c|}{$3.65(0.93)$} & \multicolumn{2}{|c|}{$3.45(1.61)$} \\
\hline GHQ-28 & \multicolumn{2}{|c|}{$22.64(15.20)$} & \multicolumn{2}{|c|}{$22.27(9.41)$} & \multicolumn{2}{|c|}{$27.50(11.88)$} & \multicolumn{2}{|c|}{24.36 (14.93) } \\
\hline
\end{tabular}

$\mathrm{ITT}=$ Intention to Treat, full sample $(n=44 ; \mathrm{NAC}=22$, Placebo= 22); PP= Per Protocol $(n=35 ; \mathrm{NAC}=20$, Placebo= 15); ( )= Standard Deviation; YBOCS= Yale-Brown Obsessive Compulsive Disorder Scale (YBOCS); Obsessions/Compulsions= YBOCS subscales; HAMA= Hamilton Anxiety Rating Scale (HAMA); MADRS= Montgomery-Asberg Depression Rating Scale; CGI-S/I= Clinical Global Impressions (Severity/Improvement); GHQ-28= General Health Questionnaire; + Significant interaction for per protocol analysis $p=0.013$ (all over results non-significant); ITT data displayed for HAMA, MADRS, CGI-S/I and GHQ-28 
Figure 2. Results on YBOCS (per protocol)

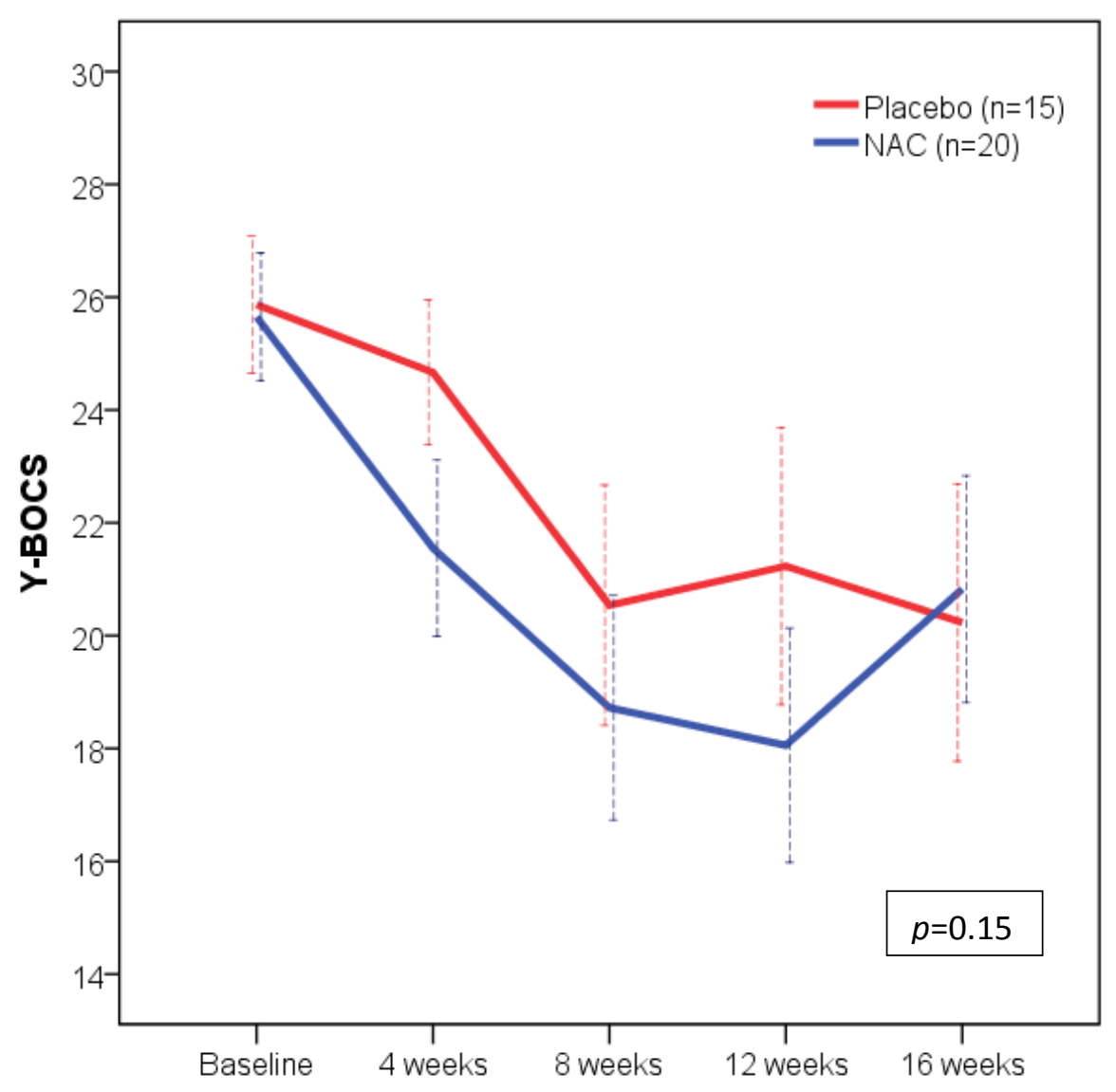

YBOCS $=$ Yale-Brown Obsessive-Compulsive Scale; NAC = N-Acetyl Cysteine

* All Group X Time interactions using MMRM across all time points revealed $p>0.05$;

( ) = Standard Deviations \# Week-4 taken as baseline 
Figure 3. Results on YBOCS Compulsions subscale (per protocol)

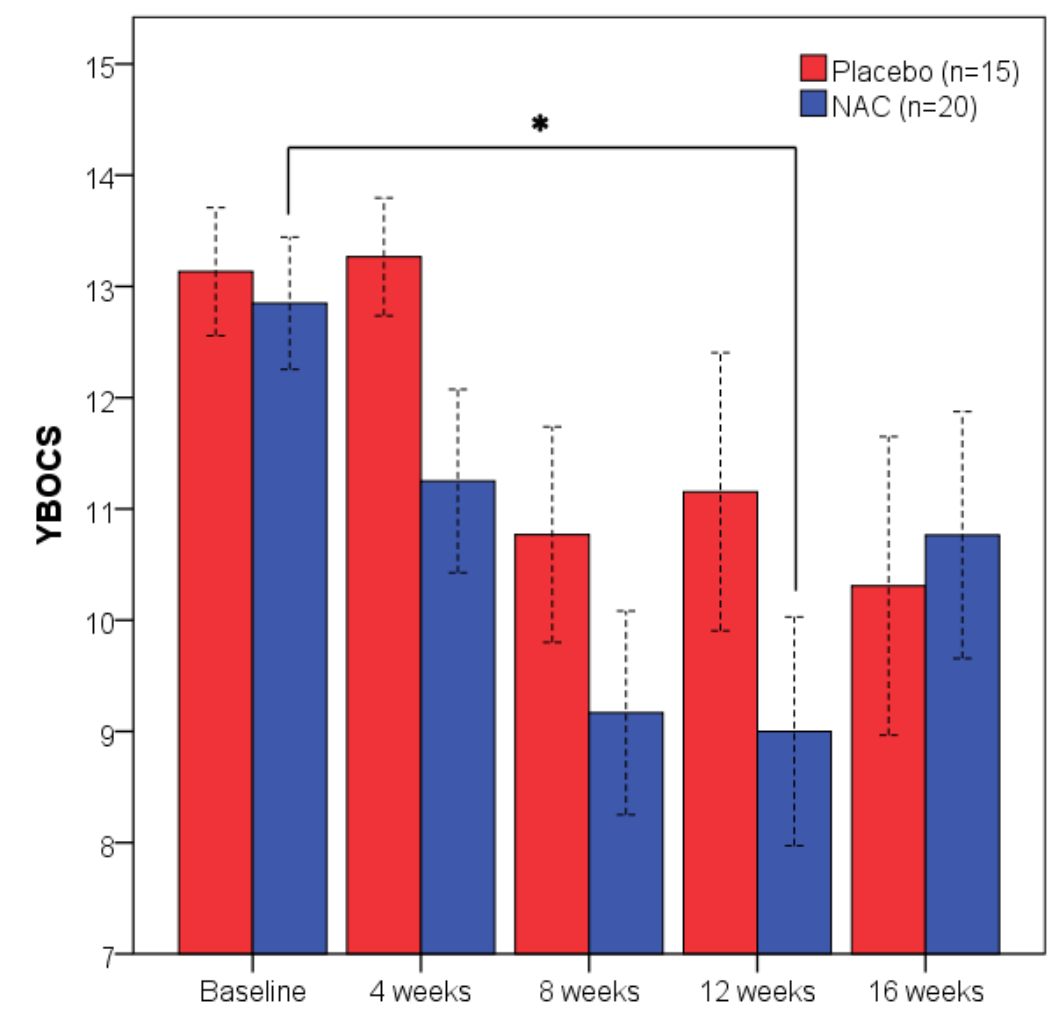

YBOCS = Yale-Brown Obsessive-Compulsive Scale

* Group X Time interaction $p=0.013$; significance at Week 12 . 